\title{
PROPOSTA DE EXPERIMENTO PARA ANALISE DA PERCEPÇÃO DE PILOTOS EM RELAÇÃO A ALERTAS EM COCKPITS DE AERONAVES
}

\author{
FELIPE M.S. TURETTA ${ }^{1}$, LUÍS GONZAGA TRABASSO ${ }^{2}$,
}

\author{
1. Embraer SA, São José dos Campos, São Paulo, 12227-901, Brasil \\ felipe.turet taembraer.com.br
}

\author{
2. Instituto Tecnológico de Aeronáutica, São José dos Campos, São Paulo, 12228-900, Brasil \\ gonzaga@ita.br
}

\begin{abstract}
Cockpit design in modern airplanes is a subject with a constant increase in its complexity, as new technologies are being incorporated in aircrafts and the pilots are becoming system managers instead of just pilots depending only on physical and coordination skills. The more complex the aircraft the more information the pilot needs to manage the flight correctly and thus more important is that the cockpit design be done in such a way to filter which information the pilot needs to know at each moment in flight, avoiding any information overload that could cause an event where an important piece of information goes unnoticed. In this scenario there is one kind of information that has the maximum importance, which are those related to failure or abnormal systems conditions, that when displayed require crew action in response to the failure, avoiding damage to the aircraft or its occupants. Thus it is desired to study how well can a pilot perceive and consequently react to different kinds of alerts presented to him in flight.
\end{abstract}

Keywords— Hybryd Systems-Man-Machine-Interface, Human Factors, Cockpit Design.

Resumo- O projeto de cockpits em aviões modernos é um assunto cuja complexidade vem aumentando constantemente a medida em que novas tecnologias vão sendo incluídas nas aeronaves e os pilotos se tornam cada vez mais gerentes de sistemas e menos pilotos dependentes de habilidades físicas e de coordenação. Quanto mais complexa a aeronave, mais informação o piloto necessita para gerenciar corretamente o vôo, e mais importante se torna o projeto do cockpit, displays e controles; de forma a filtrar corretamente as informações as quais o piloto precisa saber em cada momento do vôo, evitando uma sobrecarga de informação desnecessária, o que aumentaria a chance da informação relevante passar desapercebida no momento de necessidade. Neste cenário, existe um tipo de informação que tem a máxima importância; aquelas informações ou alertas que estão ligadas a condições de falha na aeronave e que, caso mostradas, deverão levar o piloto a tomar ações e medidas para responder a falha, evitando danos a aeronave ou seus ocupantes. Logo, deseja-se estudar o quão bem um piloto percebe, e conseqüentemente reage, a diferentes tipos de alertas apresentados a ele durante o vôo.

Palavras-chave—Sistemas Híbridos Homem-Máquina, Fatores Humanos, Projeto de Cockpits.

\section{Introdução}

O projeto de cockpits em aviões modernos é um assunto cuja complexidade vem aumentando constantemente a medida em que novas tecnologias vão sendo incluídas nas aeronaves e os pilotos se tornam cada vez mais gerentes de sistemas e menos pilotos dependentes de habilidades físicas e de coordenação. Quanto mais complexa a aeronave, mais informação o piloto necessita para gerenciar corretamente o vôo, e mais importante se torna o projeto do cockpit, displays e controles; de forma a filtrar corretamente as informações as quais o piloto precisa saber em cada momento do vôo, evitando uma sobrecarga de informação desnecessária, o que aumentaria a chance da informação relevante passar desapercebida no momento de necessidade.

Neste cenário, existe um tipo de informação que tem a máxima importância; aquelas informações ou alertas que estão ligadas a condições de falha na aeronave e que, caso mostradas, deverão levar o piloto a tomar ações e medidas para responder a falha, evitando danos a aeronave ou seus ocupantes.

Inicialmente parece óbvio que alertas ligados a tais tipos de falhas deveriam ser projetados para te- rem "visibilidade máxima", utilizando alertas visuais no campo primário de visão e redundâncias sensoriais como alertas aurais e até mesmo tácteis dependendo das circunstâncias. Porém em muitos momentos, certas condições de falha não são previstas no projeto inicial da aeronave, sendo detectadas durante a vida em serviço e necessitando análise. Em alguns casos dependendo dos alertas disponíveis para uma certa condição falha, e das características e consequiências da mesma, pode ser necessário (ou não) reprojetar ou modificar a aeronave. Neste relatório estudaremos as características de cada alerta, e o quão bem um piloto é capaz de percebê-lo para tomar as ações necessárias. O objetivo de tal análise será servir como informação para ajudar a tomada de decisão, se uma modificação na aeronave será necessária ou não. Outros aspectos necessários para esta tomada de decisão (como gravidade da falha, probabilidade etc) não serão abordados neste trabalho.

\section{Formulação do problema}

Primeiramente deve-se definir 2 parâmetros; as características de uma alerta e os fatores que afetam a sua percepção. O elemento humano é extremamente importante neste problema, e muitas características da percepção não são contínuas ou exatas; sendo 
assim, serão definidos os parâmetros a serem no experimento, e os mesmos serão tratados como variáveis qualitativas.

Além disso, algumas características de uma alerta podem ser "Diretas" ou "Indiretas" em relação a uma falha. Chamam-se de diretas, aquelas indicações que ligam direta e exclusivamente a aquela falha, são óbvias e "inconfundíveis" caso percebidas (ex. uma mensagem de EICAS "ENGINE FAILURE"). Chamam-se de indiretas aquelas informações que, se percebidas, ainda necessitarão de alguma inferência do piloto ou combinação com alguma outra informação para que ele chegue a conclusão de que uma determinada falha ocorreu; não são necessariamente óbvias (ex1: Um indicador de velocidade errado, necessita comparação com outros para determinarmos se há uma evento de velocidade errônea; uma indicação de quantidade de combustível diferente em dois tanques que necessita uma comparação com um valor calculado para se determinar se há um vazamento).

- Características do alerta:

○ Indicação visual

- Ausente

- Direta - Campo de visão central

- Direta - Campo de visão paracentral

- Direta - Campo de visão periférica

- Indireta - Campo de visão central

- Indireta - Campo de visão paracentral

- Indireta - Campo de visão periférica

- Escondida (em páginas secundárias de sistemas ou FMS)

○ Indicação Aural

- Presente - Direta (Ex, “CABIN” ou "STALL”)

- Presente - Indireta (Ex, "Chimes" ou "bells")

- Ausente

○ Indicação Táctil

- Presente - Direta (Ex, Stick Shaker)

- Presente - Indireta (Ex,Vibração da fuselagem, tendências de rolamento)

- Ausente

- Fatores que afetam a percepção:

- Fatores ligados ao piloto (treinamento, alimentação, sono etc)

- Carga de trabalho da fase de vôo
- Decolagem e subida

- Cruzeiro

- Descida e pouso

- Tipo de operação

- Single Pilot

- Dual Pilot - Flying

- Dual Pilot - Monitoring

- Tipo de voo

- Manual "Head-Up"

- Manual "Head-Down"

- PA "Head-Up"

- PA "Head-Down"

- Outros (horário do dia, controle de tráfego aéreo, )

\section{Análise Dos Possíveis Fatores E Respectivos Ní- veis}

Existem muitos fatores com potencial para afetar a percepção de um piloto, logo para projetar um experimento com resultados válidos, deve-se chegar a uma simplificação dos fatores que possam ser analisados e produzam resultados satisfatórios. Essa análise foi feita usando o modelo de estudo de fatores humanos SHELL, porém a análise não será mostrada neste artigo por ser extensa e não ser o foco deste artigo. Após a análise SHELL 3 fatores de interesse foram identificados; respectivamente, Posição do alerta, "Highlights" do alerta e "Workload", e 1 bloco: piloto.

\section{Análise Das Condições Experimentais E Escolha Do Tipo De Experimento}

Para avaliar a percepção do piloto, ele deverá ser exposto a um ambiente operacional próximo do real em um simulador de vôo. Posteriormente serão simuladas as falhas e será medido o tempo de percepção dos alertas. Porém a "curva de aprendizado" do piloto sujeito ao teste (percepção do piloto de sobre o que está sendo medido) é um fator que pode contaminar os resultados do experimento, sendo assim, expor o mesmo piloto a diversos pontos de ensaio é um problema a ser tratado. Da mesma forma, queremos "blocar" o piloto para que a diferença entre um piloto e outro também não contamine o resultado. $\mathrm{Ou}$ seja, é necessário utilizar algum método para diminuir o número de testes com cada piloto, em quanto ao mesmo tempo, fazendo todos os pontos com cada um dos pilotos, para que possamos eliminar a diferença entre os pilotos dos resultados. 
Juntando o explanado acima com o fato do fenômeno ser pouco conhecido (não se sabe o quanto os fatores afetam a percepção), parece ser viável o uso de um projeto de experimento $2^{3}$, onde os três fatores de interesse definidos acima serão variados em dois níveis. Isso dará uma idéia inicial do fenômeno, nos possibilitando uma expansão do experimento posteriormente se for necessário.

Um experimento $2^{3}$ tradicional resultaria em 8 rodadas para cada um dos blocos. Isso quer dizer que cada um dos pilotos seria exposto a oito falhas diferentes de modo a compor os blocos experimentais. Qualitativamente falando, este número parece elevado, passando a impressão que nas últimas falhas, o piloto já estará esperando a falha acontecer e procurando "avidamente" pelas indicações. Isto é um comportamento indesejado para o resultado do experimento, pois provavelmente os tempos obtidos nas últimas rodadas serão relativamente menores que os obtidos nas primeiras rodadas, para os mesmos alertas. Sendo assim seria desejável diminuir ainda mais o número de rodadas por piloto. Uma maneira de fazer isso é usar um experimento fracionado, o que aponta para um experimento $2^{3}-1$ de resolução III. Tal experimento necessitará de apenas 4 rodadas por bloco, quantidade mais plausível de não contaminar a atenção do piloto aos alertas. Isso resultará em um efeito indesejado, que será a perda da capacidade de prever o efeito da relação entre os fatores (Posição $\mathrm{x}$ "Highlights" x "Workload"), porém neste estágio da pesquisa, será aplicado o conceito da "Navalha de Ockham", e considerado que os efeitos majoritários serão devidos aos fatores isolados.

Serão escolhidos de maneira arbitrária os níveis positivos da tabela do experimento fatorial completo ( $\mathrm{I}=\mathrm{ABC}$, onde $\mathrm{A}=$ Posição, $\mathrm{B}=$ "Highlights" e $\mathrm{C}=$ "Workload")

\section{Descrição Simplificada Do Procedimento Expe- rimental}

Por motivos operacionais, é interessante posicionar os pontos de medição todos dentro de um mesmo vôo simulado. Desta forma o piloto sujeito ao teste realizará apenas um vôo no qual será possível tirar todas as medidas, tornando o experimento mais rápido, barato, aumentando veracidade da simulação e aproximando o teste das condições reais de um vôo. Logo os pontos de medição serão encaixados dentro de um ciclo de Decolagem-Subida-Cruzeiro-DescidaPouso.

Considerando o experimento fatorial $2^{3}-1$ de resolução III, existirão quatro pontos de teste, onde cada fator terá dois pontos em "High" e dois pontos em "Low". O fator "Workload" está altamente atrelado a fase de vôo, logo é conveniente fazer organizar esses pontos de acordo. Assim serão medidos os pontos de "Workload-High" na decolagem e no pouso. Os pontos de "Workload-Low" deverão ser feitos durante o cruzeiro. Excluindo essa restrição, a ordem dos alertas será randomizada no vôo, das quatro seguintes formas possíveis:

Possibilidade 1: c-a-b-abc

Possibilidade 2: c-b-a-abc

Possibilidade 3: abc-a-b-c

Possibilidade 4: abc-b-a-c

Tabela 1: Experimento fatorial $2^{3}-1$ de resolução III, com I=ABC

\begin{tabular}{|c|c|c|c|}
\hline Run & $\mathrm{A}=$ Posição & $\mathrm{B}=$ "Highlights" & $\mathrm{C}=$ "Workload" \\
\hline $\mathrm{a}$ & + & - & - \\
\hline $\mathrm{b}$ & - & + & - \\
\hline $\mathrm{c}$ & - & - & + \\
\hline $\mathrm{abc}$ & + & + & + \\
\hline
\end{tabular}

Os fatores Posição e "Highlights" não são dependentes da fase de vôo, logo serão distribuídos de acordo com a conveniência das falhas simuladas disponíveis no simulador de vôo. Em anexo (Figuras $1 \mathrm{e}$ 2) mostramos como serão variados os fatores Posição e "Highlights".

Como não existe no cockpit, a mesma indicação em displays diferentes, dependendo do fator posição, será usado um alerta diferente, para variar o fator "Highlight", conforme mostrado na figura 3 (em anexo).

Definido o ambiente experimental e o método, o procedimento (apenas em linhas gerais) será o seguinte:

a. Receber o piloto e fazer um briefing sobre o vôo (Vôo de A para B, pedir que voe com o AP-OFF, caso perceba qualquer coisa anormal, avisar o experimentador, etc)

b. Levar o piloto ao simulador, e ligar a câmera para gravar o vôo

c. Escolher uma das possibilidades de seqüência de falha aleatoriamente

d. Pedir ao piloto para iniciar o vôo

e. Executar as falhas conforme ordem definida

f. Ao final do vôo, analisar a gravação e determinar os tempos de percepção.

g. Após 5 experimentos:

i. Fazer a ANOVA dos resultados e avaliar os resíduos.

ii. Avaliar o tamanho da amostra, utilizando curvas OCC. A força desejada para o teste é de 0,95.

iii. Caso o teste não tenha a força desejada, continuar a realizar repetições até chegar à força desejada.

\section{Estratégia Para Analise Dos Resultados E Po- tenciais Experimentos Posteriores}

Tendo definido o projeto de experimento acima, é importante planejar a estratégia para a análise dos dados e os experimentos posteriores. Isto, pois premissas foram assumidas para a definição do procedimento inicial, e após os primeiros experimentos, será possível analisar os dados e verificar se as premissas 
foram válidas ou não. E independente da validade das premissas, pode ser que de posse de mais informações, seja conveniente mudar a estratégia experimental. Abaixo serão mostrados possíveis resultados que podem ser antevistos, e as direções planejadas para os próximos experimentos, caso tais resultados aconteçam:

a. Para análise do experimento inicial: Quando de posse de um número aceitável de repetições:

i. Analise da influência do piloto nos resultados.

1. Caso se confirme que o piloto afeta os resultados: Nenhuma mudança estratégica

2. Caso os resultados apontem que o piloto não afeta os resultados: Mudança de um experimento fracionado $2^{3}-1$ para um experimento completo $2^{3}$ com metade das réplicas. Essa mudança será interessante, pois possibilitará avaliar os efeitos das interações entre os fatores, que não mais ficarão confundidos com os efeitos principais.

ii. Caso os resultados não sejam conclusivos após 10 repetições (nenhum dos fatores afeta estatisticamente o resultado).

1. Resultados não conclusivos podem ser fruto da interação entre os fatores atrapalhando a análise. Neste caso deverão ser analisados os resultados separadamente (desconsiderando um dos fatores que suspeite-se que influência menos)

2. Independente da influência do piloto nos resultados, fazer alguns pontos do outro para completar o experimento fatorial (repetindo pilotos ou não, dependendo da avaliação dos resultados iniciais), e tentar verificar se é possível obter resultados melhores considerando a interação.
3. Realizar alguns pontos variando outros fatores (hora do dia, etc) que podem estar contaminando o resultado, e verificar se eles deveriam estar blocados.

b. Para evolução do experimento: Quando se chegar a resultados aceitáveis:

1. Incluir o fator AP-ON-OFF, ou Single-Pilot x Dual-Pilot, de interesse, evoluindo o experimento para um $2^{4}-1$ usando $\mathrm{I}=-\mathrm{ABCD}$, desta forma metade do experimento já estará pronto. Para fazer isso ou serão usados os mesmos pilotos do primeiro experimento (mantendo o bloco, mas expondo o experimento ao aprendizado do piloto), ou serão usados pilotos diferentes (perdendo a blocagem). Tal decisão deverá depender dos resultados obtidos na primeira rodada de experimentos.

2. Realizar o experimento em uma aeronave diferente, para avaliar os efeitos da aeronave.

3. Utilizar "Highlights" diferentes (Âmbar x Vermelho - ponto central no fator "Highlight").

4. Utilizar posições diferentes (EICAS x MCDU - Ponto central no fator "Posição")

5. Outras.

\section{Conclusão}

Concluí-se desta proposta de experimento que é viável fazer o experimento, e que ele apresenta boas possibilidades de expansão para inclusão de novos fatores no futuro. Porém apenas após realizar os primeiros experimentos será possível saber se a estratégia foi acertada. Caso seja conclusivo, o experimento será de grande valia no projeto de novas aeronaves e como tomada de decisão no momento de definir o re-projeto em aeronaves em serviço. 


\section{Referências Bibliográficas}

Hawkings, Frank H. (1993) Human Factors In Flight , Ashgate.

DOT/FAA/AR-08/45. (2008) Development of a Scoring Algorithm for Flight Crew Intervention Credit in System Safety Assessments

Montgomery Douglas C. (1997). Design and Analysis of Experiments, John Wiley \& Sons. 


\section{PROPOSTA DE EXPERIMENTO PARA ANALISE DA PERCEPÇÃO DE PILOTOS EM RELAÇÃO A ALERTAS EM COCKPITS DE AERONAVES}

\section{ANEXO}

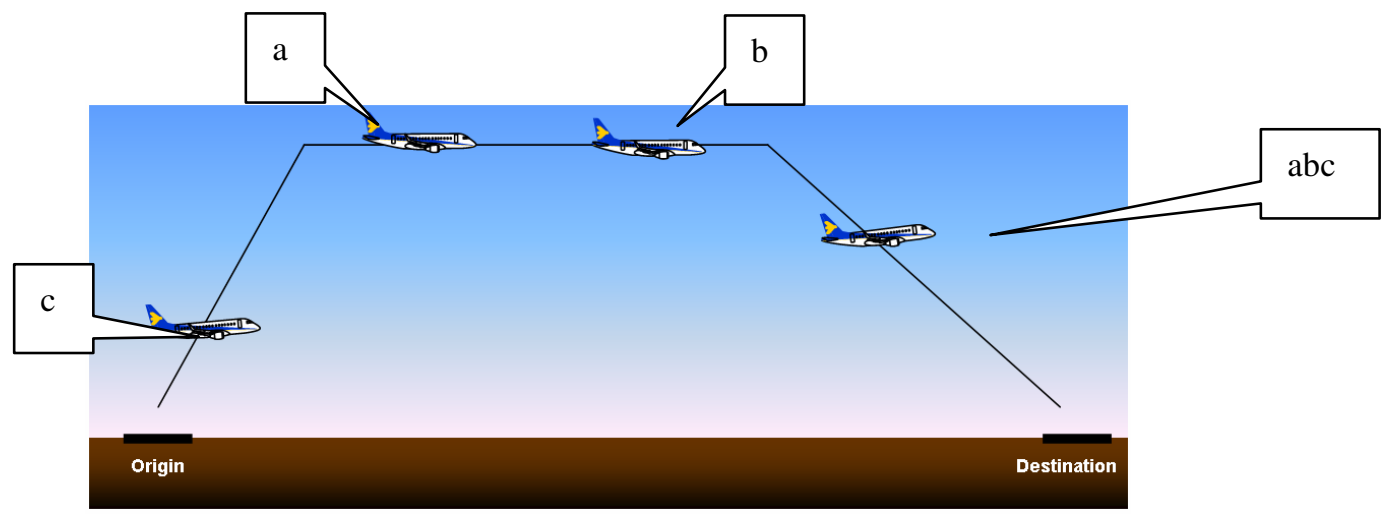

Figura 1: Sequiência de um vôo, considerando a possibilidade 1

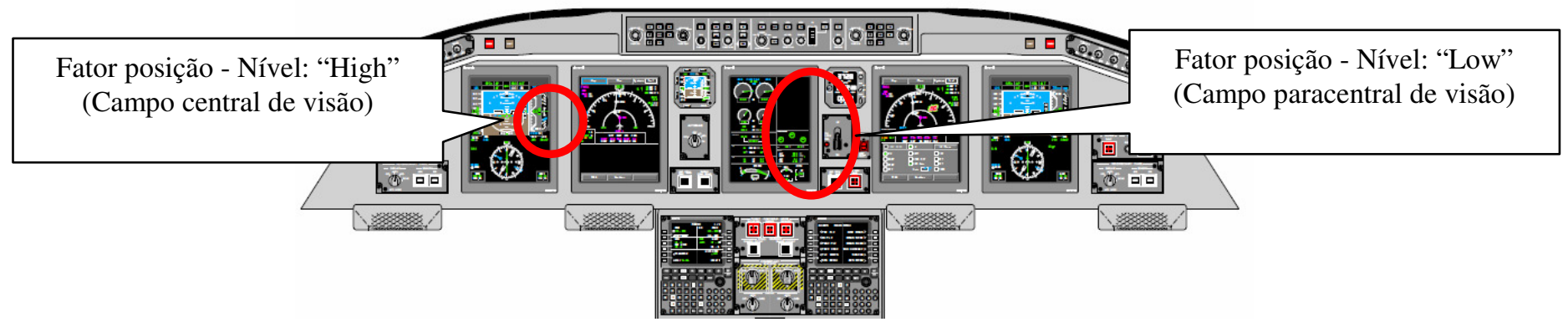

Figura 2: Variação do fator posição

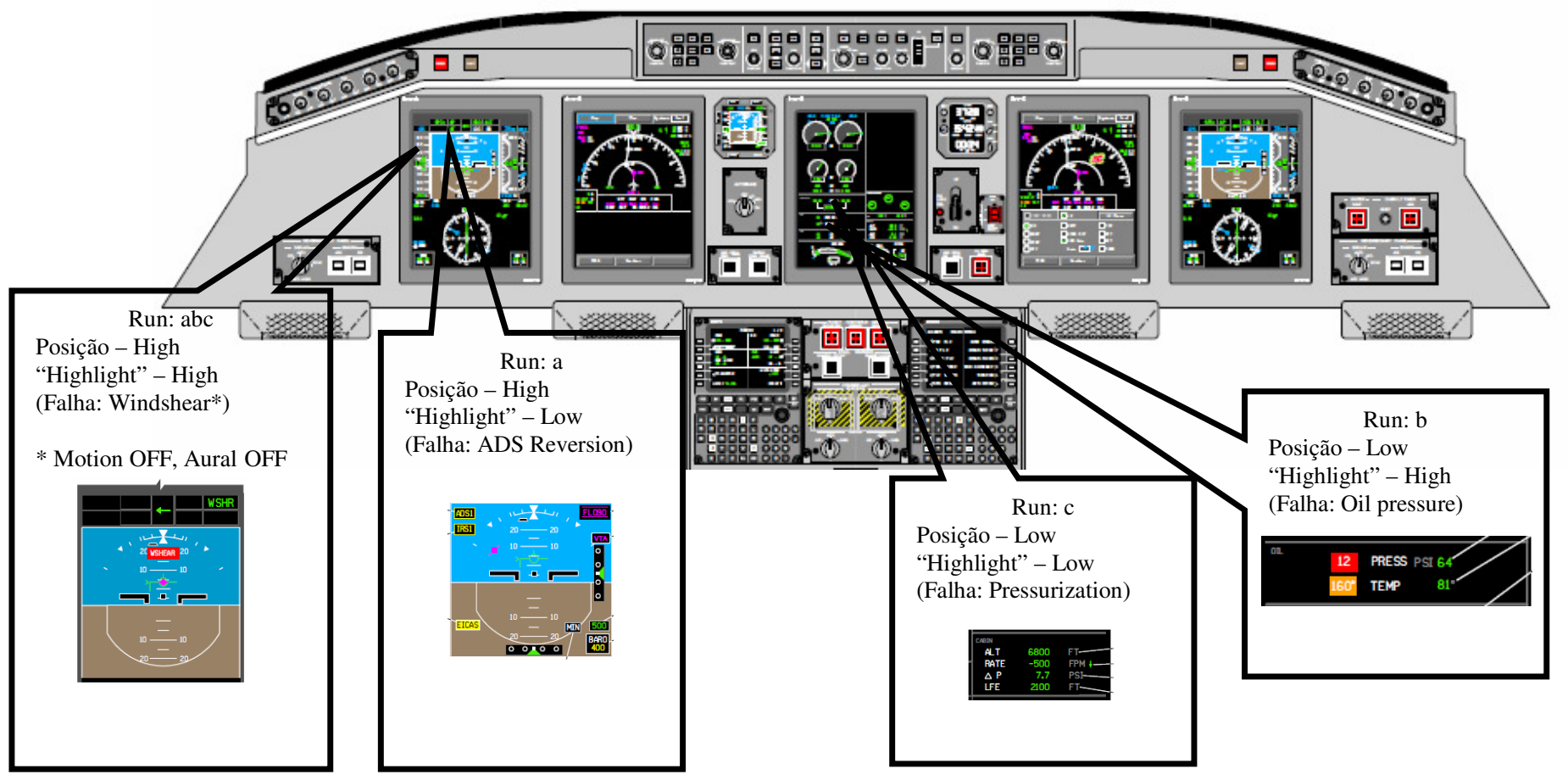

Figura 3 : Variação dos fatores Posição e "Highlight" 